\title{
Celiac disease and dysfunctional uterine bleeding; the efficiency of gluten free diet
}

\author{
Ehsani-Ardakani MJ ${ }^{1}$, Fallahian $\mathrm{M}^{2}$, Rostami K ${ }^{3}$, Rostami-Nejad $\mathrm{M}^{1}$, Lotfi $\mathrm{S}^{2}$, Mohaghegh-Shalmani $\mathrm{H}^{1}$, \\ Dabiri $\mathrm{R}^{1}$, Norouzinia $\mathrm{M}^{1}$, Azizpour-Shoobi $\mathrm{F}^{2}$, Zali $\mathrm{MR}^{1}$
}

Gastroenterology and Liver Diseases, Research Center, Shahid Behesti University of Medical Sciences,

Tehran, Iran. m.rostamii@gmail.com

\begin{abstract}
The aim of this study was to investigate the relation between Celiac disease (CD) and unexplained dysfunctional uterine bleeding (DUB) in celiac women. The celiac patients were selected from women who were referred to celiac department. Controls were selected from those women without any signs of celiac disease and matched with age. Meanwhile, a trained physician was ready to explain the study, and then in case of their allowance, a questionnaire was completed by the physician. $24 \%$ of celiac women reported a past history of at least one menstrual cycle disorder vs $10 \%$ of controls reported these problems $(p=0.038)$ and higher percentage of unexplained DUB has been observed in celiac women. All celiac patients were undertaking gluten free diet for at least 3 months and the celiac patients who reported the history of DUB were again interviewed for any signs of unexplained DUB. From 12 celiac women with DUB, 10 patients reported no more unexplained DUB after getting gluten-free diet (83.3\%). The occurrence of a significant correlation between CD and DUB suggests the possibility of considering $C D$ as one of the potential causes of abnormal uterine bleeding. Therefore, celiac disease must be seriously considered in the screening of patients with reproductive disorders (Tab. 2, Ref. 23). Text in PDF www.elis.sk.

Key words: celiac disease, dysfunctional uterine bleeding, gluten free diet.
\end{abstract}

Celiac disease (CD) is an autoimmune disorder characterized by gluten sensitivity in genetically susceptible individuals $(1,2)$. $\mathrm{CD}$ causes small bowel inflammation and is associated with increased small bowel permeability. It is associated with both intestinal and several extra-intestinal manifestations/complications, including infertility and adverse pregnancy outcomes (2-4). Celiac disease can affect women's reproductive life. Previous studies have suggested that women with undiagnosed $\mathrm{CD}$ have 9-fold relative risk of multiple abortions and low birth weight babies compared to women with treated $\mathrm{CD}$ (5). Aside from adverse pregnancy outcomes, CD may present with a persistent iron deficiency and abnormal weight loss during a first, but more often, second pregnancy (2).

Dysfunctional uterine bleeding (DUB) is a common problem amongst women and accounts for $20 \%$ of gynecology office visits (6). It is defined as abnormal uterine bleeding in the absence of or-

${ }^{1}$ Gastroenterology and Liver Diseases, Research Center, Shahid Behesti University of Medical Sciences, Tehran, Iran, ${ }^{2}$ Infertility and Reproductive Health Research Center, Shahid Behesti University of Medical Sciences, Tehran, Iran, and ${ }^{3}$ Dudley Group of Hospital, University of Birmingham, UK

Corresponding author: M. Rostami Nejad, Gastroenterology and Liver Diseases, Research Center, Shahid Beheshti University of Medical Sciences, Tehran, Iran.

Phone: +98.2122432518, Fax: +98.2122432517

Acknowledgments: This study has been financially supported by Research Institute of Gastroenterology and Liver disease (RIGLD), Shahid Beheshti University of Medical Sciences, Tehran, Iran and Iran National Science Foundation (INSF). This paper resulted from part of the $\mathrm{PhD}$ research of Mohammad Rostami-Nejad. ganic disease (excessively heavy, prolonged, or frequent intervals of bleeding), complications of pregnancy or systemic disease $(7,8)$.

The spectrum of abnormal uterine bleeding comprises of menorrhagia (heavy periods; blood loss $>80 \mathrm{~mL}$ ), metrorrhagia (prolonged, irregular periods), polymenorrhoea (frequent periods), oligomenorrhoea (scanty and infrequent periods), amenorrhoea (absent menstrual periods), intermenstrual bleeding and postcoital bleeding.

There is limited data on reproductive implications in Iranian women with $\mathrm{CD}$. The aim of this study was to investigate the relation between $\mathrm{CD}$ and unexplained DUB in celiac women who were referred to celiac department of Taleghani hospital. We also studied the effect of gluten-free diet on reducing the complications of DUB in women diagnosed with CD.

\section{Materials and methods}

This study was designed as a case-control study. The celiac patient's group was selected from those who were referred to celiac department of Taleghani hospital with diagnosis of celiac disease with or without dysfunctional uterine bleeding. Celiac disease was diagnosed based on positive serology and confirmation by histological assessment of small bowel biopsies. For this purpose, $5 \mathrm{cc}$ heparinized blood were be obtained. Blood sample was delivered to the laboratory within 2 hours. IgA class human anti-tissue transglutaminase (tTG) antibody using recombinant human tTG and Total serum IgA values were measured. Determinations of IgA anti-tTG antibody were carried out using a commercially available kit (AESKULISA tTG, Germany) and an enzyme-linked immuno- 
sorbent assay (ELISA) method. According to the manufacturer's indications, the result was considered positive when a value higher than $15.0 \mathrm{U} / \mathrm{ml}$ was recorded. Total serum IgA values were measured by an immunoturbidometric assay (Pars Azmoon, Iran) and serum levels below $70 \mathrm{U} / \mathrm{L}$ were considered indicative of IgA deficiency. Immunoglobulin G (IgG) tTG values were further obtained in individuals with IgA deficiency by an ELISA method, and using the commercially available kit (AESKULISA tTGG, Germany). Those serology positive for $\mathrm{CD}$ underwent biopsy specimens processing too. Histological diagnosis of CD was based on the presence of intraepithelial lymphocytes (Marsh I), crypts hyperplasia and/or villi atrophy (Marsh II to IIIc). Biopsy results were classified according to modified Marsh criteria by Rostami et al (1).

Control group was selected from those women who had not celiac disease with or without dysfunctional bleeding and referred to GI clinics and matched with age. Inclusion criteria for enrolling case and control group were:

- age range of 18-45 years old;

- celiac disease which diagnosed and confirmed by serology and biopsy after that in symptomatic cases;

- dysfunctional uterine bleeding that was diagnosed when abnormal uterine bleeding ruled out any pathology in endometrial biopsy, normal hormonal levels of prolactin and thyroid stimulating hormone (TSH) and normal pelvic ultrasound.

Exclusion criteria were:

- any pathology contributed in abnormal uterine bleeding;

- abnormal levels of TSH or prolactine; systemic disease confounding in diagnosis of celiac disease or DUB. Meanwhile, a trained physician was ready to explain the aim of study, and then in case of their allowance, a questionnaire was completed. The study was approved by the institutional ethics committees of Research Institute for gastroenterology and liver disease, Shahid Beheshti University of Medical sciences, and all participants signed a written informed consent.

\section{Results}

A total number of 50 celiac women who were diagnosed for the first time (mean age \pm SSD: $32.1 \pm 15.2$ ) and 70 healthy control (mean age \pm SD: $31.7 \pm 12.7$ ) were entered to this study. All celiac patients were new cases who diagnosed in Taleghani hospital, Gastroenterology and Liver Diseases, Research Center and referred to department of celiac disease for intervention. Celiac patients and the age-matched healthy control were interviewed for clinical and demographic factors. $59.4 \%$ of patients and $67.3 \%$ of controls were married and the mean $\pm \mathrm{sd}$ of BMI in patients was $21.3 \pm 4.4$ and in healthy controls was $21.5 \pm 5.9$ (no statistically difference). Infertility was reported in $4 \%$ of celiac women and $2.9 \%$ of controls respectively $(\mathrm{p}=0.73$ ). Also $6 \%$ of patients and $8.6 \%$ of controls reported a past history of at least one abortion $(\mathrm{p}=0.59)$ respectively (Tab. 1 ).

A higher percentage of unexplained DUB has been observed in celiac women. Twelve out of 50 cases of celiac group (24\%) had DUB but in the control group 7 out of 70 controls (10\%) had DUB what was significant ( $\mathrm{p}$ value $=0.038$ ). The logistics regression analysis indicated that the crude risk of DUB was increased
Tab. 1. Demographic factors and characteristics of Celiac and non Celiac groups.

\begin{tabular}{lccc}
\hline & $\begin{array}{c}\text { Celiac } \\
(\mathrm{n}=50)\end{array}$ & $\begin{array}{c}\text { Non-Celiac } \\
(\mathrm{n}=70)\end{array}$ & $\mathrm{p}$-Value \\
\hline Characteristics & & & \\
Age (mean $\pm \mathrm{sd})$ & $32.1 \pm 15.2$ & $31.7 \pm 12.7$ & 0.90 \\
BMI (mean \pm sd) & $21.3 \pm 4.4$ & $21.5 \pm 5.9$ & 0.89 \\
Married (\%) & $33(67.3)$ & $41(59.4)$ & 0.38 \\
DUB (\%) & $12(24.0)$ & $7(10.0)$ & 0.03 \\
Infertility (\%) & $2(4.0)$ & $2(2.9)$ & 0.73 \\
History of Abortion (\%) & $3(6.0)$ & $6(8.6)$ & 0.59 \\
\hline
\end{tabular}

Tab. 2. Association with Dysfunctional uterine bleeding and Clinical Symptoms in Celiac group.

\begin{tabular}{lccc}
\hline & $\begin{array}{c}\text { DUB } \\
(\mathrm{n}=12)\end{array}$ & $\begin{array}{c}\text { Non-DUB } \\
(\mathrm{n}=38)\end{array}$ & P-Value \\
\cline { 1 - 1 } Clinical Symptoms (\%) & & & \\
\cline { 1 - 1 } Diarrhea & $4(33.3)$ & $7(18.4)$ & 0.28 \\
Bloating & $6(50)$ & $18(47.4)$ & 0.87 \\
Heart Burn & $4(33.3)$ & $7(18.4)$ & 0.27 \\
Weight Loss & $8(66.7)$ & $12(31.6)$ & 0.03 \\
Neuse \& Vomiting & $4(33.3)$ & $4(10.5)$ & 0.06 \\
Anemia & $10(83.3)$ & $22(57.9)$ & 0.11 \\
Bone Disease & $6(50)$ & $9(23.7)$ & 0.08 \\
\hline
\end{tabular}

2.84 times for women with celiac disease compared to healthy women (95\% CI: 1.03-7.84). Also, the adjusted risk of DUB was $3.83 \%$ for celiac women according to the multivariate logistics analysis (95\% CI: 1.19-12.33).

According to the histology of CD patients who reported DUB, 1 patient was in Marsh 0; four patients were in Marsh I, two patients in Marsh II and three patients in Marsh IIIc.

We also investigated the distribution of gastrointestinal complications and other diseases (which had been registered in CD patient's documents) with DUB (Table 2). The results indicated that there was a significant association between DUB and weight loss $(\mathrm{P}=0.03)$, which means that patients with lower weight were experiencing a higher risk of unexplained DUB. Other symptoms and diseases were not significant.

All celiac patients were undertaking gluten free diet for at least 3 months. After 3-4 months, the celiac patients who reported the history of DUB were interviewed again for report of repeated abnormal bleeding. In follow up of the patients on gluten free diet, approximately 10 out of 12 celiac cases did not complain of any abnormal uterine bleeding (83.3\%). The menarche age in patients with CD on a GFD was decreased but was higher in the untreated $\mathrm{CD}$ patients; we concluded that the age at menarche in women with $\mathrm{CD}$ was regulated by a GFD.

\section{Discussion}

This study indicated that the risk of DUB was increased in women with CD. The exact mechanism of DUB is uncertain but is thought to be caused by a dysfunction of hypothalamic-pituitaryovarian axis (9). Weight loss is another issue that has a negative impact on reproduction. On the other hand, the spectrum of gluten related disorders is widening. This is because these common systemic disorders have multifactorial etiology with a multitude 
of symptoms and complications (10). Several studies have shown that celiac disease can impair women's reproductive life eliciting delayed puberty, infertility, amenorrhea and early menopause. Some clinical and epidemiological studies have demonstrated that women with celiac disease are at a higher risk of miscarriage, low birth weight of the newborn (11-14).

In particular, celiac women have their menarche at an older age, compared to that of healthy controls, whereas the average age at menopause of celiac women has been observed to be younger than that of healthy women; in the same studies an increased frequency of cases of secondary amenorrhea has also been registered among celiac women (15).

In the study by Ferguson et al, the reproductive life of 74 celiac patients including 54 patients on normal diet and 20 under gluten free diet were studied. They found that delayed menarche and early menopause were more common in the untreated group than the controls (16). In compatibility with Ferguson et al study, Molteni et al (15) examined 54 celiac patients and 54 healthy women and detected that the mean age at menarche was significantly later in CD patients (15). In another study, the mean age of menarche in 59 girls with $C D$ was significantly higher in untreated girls compared to those who were on a GFD (17). Delayed menarche and earlier menopause in $\mathrm{CD}$ that cause a decreased number of children may be interpreted as s sub-fertility (18-20).

Previous studies suggested that women with $\mathrm{CD}$ have a higher risk of multiple abortions (5). An Italian case-control study on 62 celiac women and 186 healthy controls showed a higher percentage of menstrual cycle disorders in celiac women (21). In our study, there was no association between $\mathrm{CD}$ and infertility or abortion which may be due to small sample size. However, the results indicated that the risk of DUB was higher for women with CD.

The gluten itself, could explain the disturbances and malnutrition would worsen the disease in a consequent vicious cycle (22). Thus these reproductive disorders may be a consequence of the endocrine derangements caused by selective nutrient deficiencies (14).

The occurrence of a significant correlation between CD and DUB suggests the possibility of considering CD as one of the potential causes of abnormal uterine bleeding. Dysfunctional uterine bleeding can be treated with medical therapy, a levonorgestrel releasing IUD and hysterectomy (23).

Our results revealed that the gluten free diet could decrease the risk of DUB in CD women. Nowadays, the early diagnosis and treatment of $\mathrm{CD}$ is possible and not very costly. Therefore, celiac disease must be seriously considered in the screening and treatment of patients with reproductive disorders.

\section{References}

1. Rostami K, Kerckhaert J, Tiemessen R, von Blomberg BM, Meijer JW, Mulder CJ. Sensitivity of antiendomysium and antigliadin antibodies in untreated Coeliac disease: disappointing in clinical practice. Am J Gastroenterol 1999; 94: 888-894.

2. Khoshbaten K, Rostami Nejad M, Farzady L, Sharifi N, Hashemi SH, Rostami K. Fertility disorder associated with celiac disease in male and female; fact or fiction? J Obstet Gynaecol Res 2011; 37 (10): 1308-1312.
3. Collin P, Kaukinen K, Valimaki M, Salmi J. Endocrinological disorders and celiac disease. Endocrine Reviews 2002; 23 (4): 464-483.

4. Rostami Nejad M, Rostami K, Pourhoseingholi MA, Nazemalhosseini Mojarad E, Habibi M, Dabiri H, Zali MR. Atypical presentation is dominant and typical for coeliac disease. J Gastrointestin Liver Dis 2009; 18 (3): 285-291.

5. Ciacci C, Cirillo M, Auriemma G, Di Dato G, Sabbatini F, Mazzacea G. Celiac disease and pregnancy outcome. Amer J Gastroenterol 1996; 91 (4): 718-722.

6. Awwad JT, Toth TL, Schiff I. Abnormal uterine bleeding in the perimenopause. Int J Fertil 1993; 38: 261.

7. Pitkin J. Dysfunctional uterine bleeding. BMJ 2007; 334 (5).

8. Fraser IS, Sungurtekin U. Defining menstrual disturbances. In: Maclean A, O' Brien PMS (Eds). Study Group on Menstrual Disorders. Roy Coll Obstet Gynecol 2000; 141-152.

9. Livingstone M, Fraser IS. Mechanisms of abnormal uterine bleeding. Hum Reprod Update 2002; 8: 60-67.

10. Rostami Nejad M, Hogg-Kollars S, Ishaq S, Rostami K. Subclinical celiac disease and gluten sensitivity. Gastroenterol Hepatol Bed Bench 2011; 4 (3): 102-108.

11. Stazi AV, Mantovani A: A risk factor for female fertility and pregnancy: celiac disease. Gynecol Endocrinol 2000; 14 (6): 454-463.

12. Sher KS, Jayanthi V, Probert CSJ, Stewart CR, Maybarry JF. Infertility, obstetric and gynaecological problems in coeliac sprue. Dig Dis 1994, 12: 186-190.

13. Sher KS, Maybarry JF. Female Fertility, Obstetric and Gynecological History in Coeliac Disease. Digestion 1994; 55: 243-246.

14. Rostami K, Steegers EA, Wong WY, Braat DD, Steegers-Theunissen RP. Coeliac disease and reproductive disorders: a neglected association. Eur J Obstet Gynecol Reprod Biol 2001; 96 (2): 146-149.

15. Molteni N, Bardella MT, Bianchi PA. Obstetric and gynecological problems in women with untreated celiac sprue. J Clin Gastroenterol 1990; 12 (1): 37-39.

16. Ferguson R, Holmes GKT, Cooke WT. Coeliac disease, fertility and pregnancy. Scand J Gastroenterol 1982; 17: 65-68.

17. Rujner J. Age at menarche in girls with celiac disease. Ginecol Pol 1999; 70: 359-362.

18. Sher KS, Mayberry JF. Female fertility, obstetric and gynecological history in coeliac disease. Digestion 1994; 55: 243-246.

19. Smecuol E, Maurino E, Vasquez H, Pedreira S, Niveloni S, Mazure R, Boerr L, Bai JC. Gynaecological and obstetric disorders in coeliac disease: Frequent clinical onset during pregnancy or the pueperium. Eur J Gastroenterol Hepatol 1996; 8: 63-89.

20. Collin P, Vilska S, Heinonen PK, Hallström O, Pikkarainen P. Infertility and coeliac disease. Gut 1996; 39: 382-384.

21. Martinelli D, Fortunato F, Tafuri S, Germinario CA, Prato R. Reproductive life disorders in Italian celiac women. A case-control study. BMC Gastroenterol 2010; 10: 89.

22. Kotze LM. Gynecologic and obstetric findings related to nutritional status and adherence to a gluten-free diet in Brazilian patients with celiac disease. J Clin Gastroenterol 2004; 38 (7): 567-574.

23. Bongers MY, Mol BW, Brölmann HA. Current treatment of dysfunctional uterine bleeding. Maturitas 2004; 47 (3): 159-174.

Received February 25, 2012. Accepted April 13, 2012. 